OPEN ACCESS

Edited by:

Feng Zhu,

Zhejiang University, China

Reviewed by:

Liang Cheng,

Harbin Medical University, China

Shikha Kumari,

University of Nebraska Medical

Center, United States

*Correspondence:

Daiwei Wan

dv19880114@163.com

Qiaoming Zhi

strexboy@163.com

YeXu

xu_shirley021@163.com

tThese authors have contributed equally to this work

Specialty section:

This article was submitted to Pharmacology of Anti-Cancer Drugs,

a section of the journal

Frontiers in Oncology

Received: 20 March 2020

Accepted: 19 June 2020

Published: 31 July 2020

Citation:

Xu Z, Gao G, Liu F, Han Y, Dai C, Wang S, Wei G, Kuang Y, Wan D,

Zhi Q and Xu Y (2020) Molecular

Screening for Nigericin Treatment in

Pancreatic Cancer by

High-Throughput RNA Sequencing.

Front. Oncol. 10:1282.

doi: 10.3389/fonc.2020.01282

\section{Molecular Screening for Nigericin Treatment in Pancreatic Cancer by High-Throughput RNA Sequencing}

\author{
Zhihua Xu ${ }^{1+}$, Guanzhuang Gao ${ }^{1 \dagger}$, Fei Liu ${ }^{2}$, Ye Han ${ }^{1}$, Chen Dai ${ }^{1}$, Sentai Wang ${ }^{1}$, \\ Guobang Wei ${ }^{1}$, Yuting Kuang ${ }^{1}$, Daiwei Wan ${ }^{1 *}$, Qiaoming Zhi ${ }^{1,3 *}$ and $\mathrm{Ye} \mathrm{Xu}^{3 *}$ \\ ${ }^{1}$ Department of General Surgery, The First Affiliated Hospital of Soochow University, Suzhou, China, ${ }^{2}$ Department of \\ Gastroenterology, The First Affiliated Hospital of Soochow University, Suzhou, China, ${ }^{3}$ Department of Colorectal Surgery, \\ Fudan University Shanghai Cancer Center, Shanghai Medical College, Shanghai, China
}

Objectives: Nigericin, an antibiotic derived from Streptomyces hygroscopicus, has been proved to exhibit promising anti-cancer effects on a variety of cancers. Our previous study investigated the potential anti-cancer properties in pancreatic cancer (PC), and demonstrated that nigericin could inhibit the cell viabilities in concentration- and time-dependent manners via differentially expressed circular RNAs (circRNAs). However, the knowledge of nigericin associated with long non-coding RNA (IncRNA) and mRNA in pancreatic cancer (PC) has not been studied. This study is to elucidate the underlying mechanism from the perspective of IncRNA and mRNA.

Methods: The continuously varying molecules (IncRNAs and mRNAs) were comprehensively screened by high-throughput RNA sequencing.

Results: Our data showed that 76 IncRNAs and 172 mRNAs were common differentially expressed in the nigericin anti-cancer process. Subsequently, the bioinformatics analyses, including Gene ontology (GO) and Kyoto Encyclopedia of Genes and Genomes (KEGG) analysis, coding and non-coding co-expression network, cis- and trans-regulation predictions and protein-protein interaction (PPI) network, were applied to annotate the potential regulatory mechanisms among these coding and non-coding RNAs during the nigericin anti-cancer process.

Conclusions: These findings provided new insight into the molecular mechanism of nigericin toward cancer cells, and suggested a possible clinical application in PC.

Keywords: nigericin, high-throughput sequencing, long non-coding RNA, bioinformatics, pancreatic cancer

\section{INTRODUCTION}

Ductal adenocarcinoma of the exocrine pancreas, commonly known as pancreatic cancer (PC), is a highly aggressive malignancy with few effective therapies. At the time of diagnosis, $-20 \%$ of PC patients are considered eligible for surgery and of these, about a half undergoes successful resection (1). But unfortunately, a majority of patients with PC are diagnosed at advanced stages, at which patients can hardly receive surgical R0 resection (2) with a 5-years survival rate of 3\% and a median survival of $<6$ months (3-5). In spite of significant advances in surgical care, chemotherapy and radiotherapy, no effective systemic therapy for the aggressive pathology of this cancer is available. 
One of the reasons for the treatment failures is due to resistance to chemotherapy or radiotherapy (6). Thus, novel therapeutic agents are needed to treat PC and improve the overall survival of patients with this disease.

Up to date, standard treatments for cancer involve chemotherapy with anti-tumor antibiotic. Adriamycin, an orally administered DNA alkylating agent, has been the most potent chemotherapy applied in clinic, in addition to surgical excision. Bleomycin had been emerged as another vital chemotherapeutic agent in many types of cancer, including Hodgkin lymphoma (7), testicular cancer (8), and squamouscell carcinoma (9). In the previous study, we firstly identified salinomycin as a novel identified cancer stem cells (CSCs) killer in gastric cancer cells (10). Afterwards, we also found that salinomycin could specifically target on cisplatin-resistant colorectal cancer cells by accumulating reactive oxygen species (11). Recently, Moxifloxacin and ciprofloxacin induced cell apoptosis and S-phase arrest via ERK activation in PC (12). Similar anti-cancer influences of antibiotic on PC cells were found also in recent literatures. In 2012, Yadav et al. demonstrated that gatifloxacin possessed anti-proliferative activity against PC cell lines by causing S/G2 phase cell cycle arrest without induction of apoptosis through p21, p27, and p53 dependent pathway (13). They also investigated the effect of moxifloxacin and ciprofloxacin on survival and proliferation of PC cell lines, and found that both were able to suppress the proliferation of PC cells and induce apoptosis through the similar mechanism (12).

Nigericin is a monocarboxylic polyether antibiotic potassium ionophore that is widely used as a coccidiostatic agent in chickens (14). In 1972, the effects of nigericin on intracellular $\mathrm{pH}$, glycolysis, and $\mathrm{K}^{+}$concentration of ehrlich ascites tumor cells were firstly reported (15). Since then, emerging evidence confirmed the promising anti-cancer activity of nigericin in a variety of cancers, including prostate cancer (16), nasopharyngeal carcinoma (17), bladder cancer (18), chronic lymphocytic leukemia cells (19), and lung cancer (20). In 2004, Vaupel et al. reported that nigericin could inhibit breast cancer stem cells at least 100 times more effectively than paclitaxel in mice (21). Zhou et al. demonstrated that nigericin could suppress the colorectal cancer metastasis through inhibition of epithelial-mesenchymal transition (EMT) (22). Recently, our study explored the circular RNA (circRNA) expression profiles after nigericin exposure on PC cells through bioinformatics method, and discussed the potential function of nigericin in PC (23). However, our knowledge of nigericin, which correlates with long non-coding RNA (lncRNA) and mRNA in PC, has not been studied yet.

In this study, we attempted to ascertain the specific activities of nigericin on human PC cell lines and investigate its possible molecular mechanism in PC. The continuously varying molecules (lncRNAs and mRNAs) were displayed by the high-throughput sequencing. Through analyzing the aberrant expressions of lncRNAs and mRNAs as well as their potential relationships, the molecular mechanisms of nigericin treatment on PC were discussed.

\section{MATERIALS AND METHODS}

\section{Cell Culture and Reagents}

Human PC cell lines (PANC-1) were purchased from Shanghai Institute of Biochemistry and Cell Biology at the Chinese Academy of Sciences (Shanghai, China). Cells were cultured in Dulbecco's Modified Eagle Medium (DMEM, Gibco) supplemented with $10 \%$ fetal bovine serum (FBS, Gibco) at $37^{\circ} \mathrm{C}$ in a humidified incubator containing $5 \% \mathrm{CO}_{2}$. Cells were in the logarithmic phase of growth for all experiments. Nigericin was purchased from Sigma Aldrich (USA). The stock solutions (100 $\mathrm{mmol} / \mathrm{L}$ ) were prepared with dimethyl sulfoxide (DMSO) and stored at $-20^{\circ} \mathrm{C}$.

\section{High-Throughput RNA Sequencing Analysis}

PANC-1 cells were exposed to a proper concentration of nigericin $(5 \mu \mathrm{mol} / \mathrm{L})$ according to the results of $50 \%$ inhibitory concentration (IC50) for different time periods $(0,8,16$, or $32 \mathrm{~h}$ ), and then total RNA was extracted from cells, respectively. The quantity and integrity of total RNAs were measured by the NanoDrop ${ }^{\mathrm{TM}}$ ND-2000 (Thermo Fisher Scientifc, Scotts Valley, CA, USA) and Agilent Bioanalyzer 2100 (Agilent Technologies, Santa Clara, CA, USA), respectively. lncRNAs and mRNAs were quantitatively analyzed by Shanghai OE Biotech (Shanghai, China). After removal of ribosomal RNA and then constructing a library, a high-throughput RNA sequencing was performed. The clean reads were aligned to the reference genome by Bowtie2 (http://bowtie-bio.sourceforge.net/bowtie2/manual. shtml). For unmapped reads, the junctions were picked out using back-splice algorithm. Finally, lncRNAs and mRNAs were verified with software developed by Shanghai OE Biotech, which were considered as the reference sequence for further analysis.

\section{Differentially Expressed IncRNA and mRNA Screen and Clustering Analysis}

Differentially expressed lncRNAs and mRNAs were detected by the negative binomial distribution test based on the DESeq package. These lncRNAs and mRNAs with statistical significance were screened with $p<0.05$, false discovery rate (FDR) $<0.05$ and fold change (FC) more than 2.0. Difference integration analysis (Venn analysis) was used to show the often characteristic elements among these 3 compared groups ( 0 vs. $8 \mathrm{~h}, 0$ vs. $16 \mathrm{~h}$, 0 vs. $32 \mathrm{~h}$ ). The common differentially expressed lncRNAs and mRNAs were showed in pies with different colors. The nonsupervised hierarchical clustering of the differentially expressed lncRNAs and mRNAs was used in the form of heat map to display the expression patterns of the differential lncRNAs and mRNAs between different groups.

\section{Quantitative Real-Time Polymerase Chain Reaction (qRT-PCR) Validation}

Total RNA from cell lines was extracted using Trizol solution (Invitrogen, USA) and converted into cDNA by using M-MLV reverse transcriptase (Invitrogen, USA). The quantities and qualities of isolated RNAs were evaluated using absorbance 
measurements at 260 and $280 \mathrm{~nm}$. Then reverse transcription (RT) was performed in a $20 \mu \mathrm{l}$ reaction system using the ReverAid First Stand cDNA Synthesis (Thermo Scientific, Mountain View, CA, USA). RT-PCR with SybGreen I (Generay Bio Co., Shanghai, China) was performed using the 7500 real-time PCR system (Applied Biosystems, Hayward, CA, USA) with the follow program: initial denature at $95^{\circ} \mathrm{C}$ for $10 \mathrm{~min}$, followed by 40 cycles of $95^{\circ} \mathrm{C}$ for $10 \mathrm{~s}$ and $60^{\circ} \mathrm{C}$ for $60 \mathrm{~s}$. $\beta$-actin was used as control. Results were harvested in three independent wells. The sequences of primers were listed as follows: LINC00667:6 (F: 5'CCCGACTTTTTGATGCAGGC3'; R: 5'CCCGACTGTTTCCTACCCAC3'), Lnc-HMGN1-1:12 (F: 5'GATCATGGCTCTCTCTGCCA3'; R: 5'AGCTGTTACATA CGGCCCAC3'), Lnc-LRRC24-2:1 (F: 5'GATTCGCTGGAC GATCGCA3'; R: 5'CCTGTAAAGGGAACGCGTCA3'), LncAC007952.1.1-3:1 (F: 5'GCGAGAAAGGTTTTCGCCTC3'; R: $\quad 5^{\prime}$ ACAATAGGAGGTGCCACACA3'), Lnc-CCNB1IP11:2 (F: 5'TGTCCCTTGGGAAGGTCTGA3'; R: 5'CCCG TTCTCTGGGAACTCAC3'), GADD45A (F: 5'GAGAGCAGAA GACCGAAAGGA3'; R: 5'CACAACACCACGTTATCGGG3'), HBP1 (F: 5'TCATCACCATTGGAAGGAGGA3'; R: 5'TTGCAC CATCCCAAATCATCA3'), SESN2 (F: 5'AAGGACTACC TGCGGTTCG3'; R: 5'CGCCCAGAGGACATCAGTG3'), KIF20A (F: 5'TTGAGGGTTAGGCCCTTGTTA3'; R: 5'GTCCTTGGGTGCTTGTAGAAC3'), TOP2A (F: 5'ACCAT TGCAGCCTGTAAATGA3'; R: 5'GGGCGGAGCAAAATAT GTTCC $3^{\prime}$ ), and $\beta$-actin (F: 5'CCTGTACGCCAACACAGTGC3'; R: 5'ATACTCCTGCTTGCTGATCC3').

\section{Gene Ontology (GO) and Kyoto Encyclopedia of Genes and Genomes (KEGG) Pathway Analysis}

$\mathrm{GO}$ analysis was conducted to construct meaningful annotations of genes and gene products in a wide variety of organisms through DAVID database (http://david.abcc.ncifcrf.gov). Our GO analysis provided the ontology of defined terms which represented gene product properties, and covered three domains: cellular components, biological process and molecular function. The top 10 enriched GO terms, which were derived from the common differentially expressed mRNAs and ranked by enrichment score, were presented. KEGG pathway analysis was also adopted to map differentially expressed mRNAs in different biological pathways. The top 20 enriched pathways among the four groups ranked by enrichment score were calculated and shown.

\section{IncRNA-mRNA Co-expression Network}

To elucidate the potential functions of differentially expressed lncRNAs and explore the relationships between common differentially expressed lncRNAs and mRNAs, the lncRNAmRNA co-expression network was constructed. For each differentially expressed IncRNA, we calculated the Pearson Correlation of its expression value with the expression value of each differentially expressed mRNA. It was considered to be correlated when the $P$-value of the correlation coefficient of lncRNAs and mRNAs' expression value was not higher than 0.05 , and the absolute value of correlation was not $<0.7$. A total of 66 lncRNAs and mRNAs were selected to generate the network map.

\section{Cis- and Trans-Regulation Predictions}

As previous studies defined, a cis-regulator is the one that exerts its functions on the neighboring genes which were located at the same chromosome. IncRNAs are showed that they can regulate gene expressions in a cis-manner $(24,25)$. The cis-regulation regions in this study were identified by the following procedures. For each common differentially expressed lncRNA, we identified the mRNAs as "cis-regulated mRNAs" when: (1) the mRNAs loci were within $100 \mathrm{k}$ windows up- and downstream of the given lncRNA. (2) the Pearson Correlation of lncRNA-mRNA expression was statistically significant ( $p$-value of correlation $\leq 0.05$ ).

For trans-regulation prediction, we focused on the manner that lncRNAs played their functions via transcription factors (TFs). The TF-lncRNA and TF-lncRNA-gene network were constructed, respectively. For each differentially expressed lncRNAs, the coding genes co-expressed with them were calculated, and the significance of the gene enrichment in each TF entry was calculated using the hypergeometric distribution test method. The result of the calculation returned a $p$ value that was enriched for significance. A small $p$-value indicated that gene has been enriched in the TF entry. We calculated the intersection of lncRNAs co-expressed gene sets with target gene sets of transcription factor/chromatin regulated complex, and calculated the degree of enrichment of the intersection through hypergeometric distribution method. Then we obtained the TFs which were significantly associated with lncRNAs, and identified possible transcription factor/chromatin regulated factors that might play a combined regulatory role with lncRNAs. Subsequently, we used the analysis results of hypergeometric distribution to visualize the network diagram. Through the hypegeometric distribution calculation, each lncRNA got multiple TF-lncRNA relationship pairs, and each TFlncRNA pair was the results of enrichments of multiple genes. According to the $p$-value from small to large sort, the top 200 lines of regulatory relationships were used to construct the TF-lncRNA binary relationship network, and the top 10 lines of regulatory relationships were applied to construct the TF-lncRNA-gene ternary relationship network.

\section{Protein-Protein Interaction (PPI) Network Construction}

The Search Tool for the Retrieval of Interacting Genes (STRING, http://string.embl.de/) database was used to construct the PPI network of the common differentially expressed mRNAs. The PPI network was subsequently visualized using Cytoscape. Confidence score $\geq 0.7$ was set as the cut-off criterion, and Molecular Complex Detection (MCODE) was conducted to screen modules of PPI network with degree cutoff $=2$, node score cutoff $=0.2, \mathrm{k}$-core $=2$, and $\max$. depth $=100$. In addition, a sub-network was constructed by selecting several candidate mRNAs. 


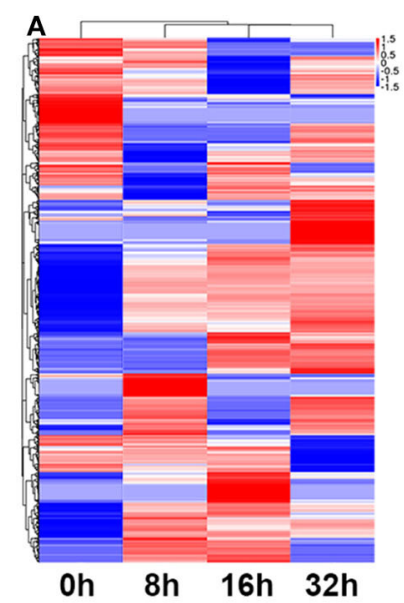

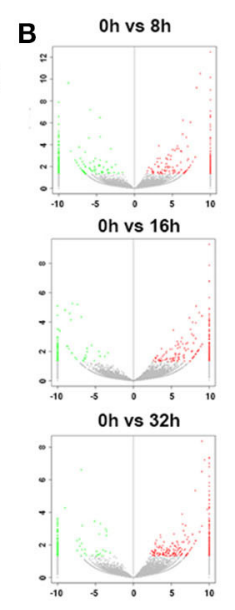

- Up-regulated

Not significant

- Down-regulated
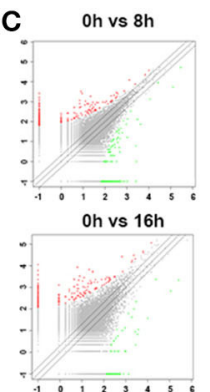

Oh vs $32 \mathrm{~h}$

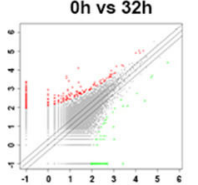

- Up-regulated Not significant

Down-regulated

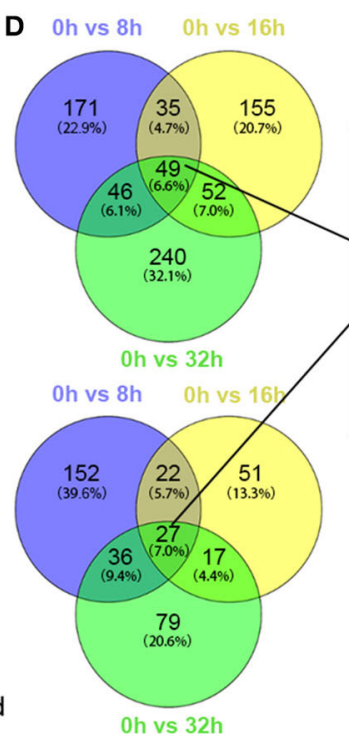

E

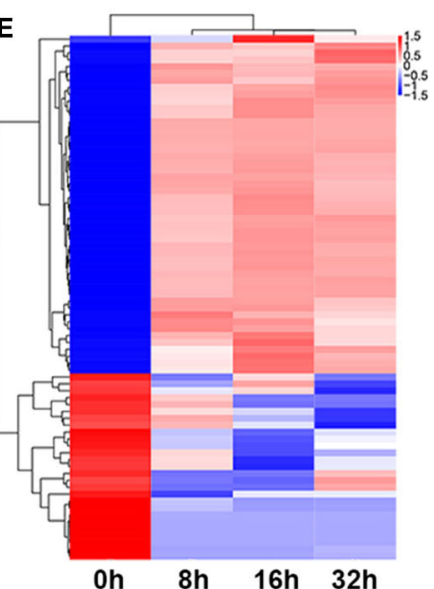

FIGURE 1 | Differentially expressed IncRNA profile by sequencing. (A) The cluster heatmap showed all differentially expressed IncRNAs at different time points (0, 8 , 16 , and $32 \mathrm{~h}$ ). (B,C) The volcano and scatter plots presented differentially expressed IncRNAs between different compared groups (0 h vs. $8 \mathrm{~h}, 0 \mathrm{~h}$ vs. $16 \mathrm{~h}, 0 \mathrm{~h}$ vs. $32 \mathrm{~h}$ ), respectively. (D,E) Venn analysis analyzed the common differentially expressed IncRNAs among the three compared groups, and described them as a cluster heatmap.

\section{Statistical Analysis}

Statistically significant differences among groups were estimated by the Student's $t$-test using SPSS 19.0 software (SPSS Inc.). $P<$ 0.05 was considered to be statistically significant.

\section{RESULTS}

\section{Differentially Expressed IncRNA and mRNA Profile by Sequencing}

The global expression profile of lncRNAs at 4 different time points $(0,8,16$, and $32 \mathrm{~h})$ was determined by a custom sequencing platform. In total, 118,314 lncRNAs were detected, and hundreds of lncRNAs showed differential expressions in each group of different time points (Figure 1A). Three compared groups were set according to the nigericin-treated time points $(0$ vs. $8 \mathrm{~h}$, 0 vs. $16 \mathrm{~h}, 0$ vs. $32 \mathrm{~h}$ ). Compared to the $0 \mathrm{~h}$ group, 538 daysregulated lncRNAs (more than 2 folds) were found in $8 \mathrm{~h}$, of which 301 lncRNAs were up-regulated and 237 ones were downregulated. Similarly, 408 lncRNAs were differentially expressed in $16 \mathrm{~h}$ group with 291 up-regulated and 117 down-regulated ones, compared to the $0 \mathrm{~h}$ group. With the change of treatment time at 32 h, 387 differential lncRNAs were up-regulated, and 159 ones were down-regulated. All differentially expressed lncRNAs with statistical significance were selected with $p<0.05$, FDR $<$ 0.05 , and FC $>2.0$ (Figures $\mathbf{1 B}, \mathbf{C}$ ). Venn analysis was used to determine the common differentially expressed lncRNAs among the three compared groups. Our data confirmed that 76 common dys-regulated lncRNAs including 49 up-regulated and 27 downregulated ones might participate in the process of nigericin damage (Figures 1D,E). To systematically predict the function of lncRNAs, lncRNA subgroup analyses were performed. These
lncRNAs were widely distributed on all chromosomes except for sex chromosome X (Figure 2A). Moreover, we adapted specific probes for these lncRNAs to classify several kinds of lncRNAs. Among these dys-regulated lncRNAs, there were $71.1 \%$ senseoverlapping, $23.7 \%$ intergenic, $1.3 \%$ intronic, $1.3 \%$ bidirectional, $1.3 \%$ antisense, and $1.3 \%$ undefined (Figure 2B).

Besides, the global expression profile of mRNAs was also observed. Among the 98,121 coding transcripts examined, hundreds of coding transcripts were differentially expressed at different time points (Figure 3A). Three compared groups were also set as described above. A total of 2,468 mRNAs exhibited significant differential expression between the 0 and $8 \mathrm{~h}$ group, in which 1,282 mRNAs were up-regulated and 1,186 ones were down-regulated. For 0 vs. $16 \mathrm{~h}$ group, 616 mRNAs were upregulated and 1,000 ones were down-regulated. Similarly, for $0 \mathrm{vs.}$ $32 \mathrm{~h}$ group, 918 mRNAs were up-regulated and 1,082 ones were down-regulated (Figures 3B,C). All the differentially expressed mRNAs with statistical significance were screened with $p<0.05$, $\mathrm{FDR}<0.05$, and FC $>2.0$. Venn analysis was also used to determine the common differentially expressed mRNAs among the three compared groups ( 0 vs. $8 \mathrm{~h}, 0$ vs. $16 \mathrm{~h}$, and 0 vs. $32 \mathrm{~h}$ ). The results showed that $172 \mathrm{mRNAs}$ were common up-regulated and 85 ones were down-regulated (Figure 3D). As shown in Figure 3E, the common dys-regulated mRNAs were evaluated by the hierarchical clustering analysis.

\section{Validation of Sequencing Data by qRT-PCR}

To ensure that our results were reliable, we assessed the expressions of $5 \operatorname{lncRNAs}$ and $5 \mathrm{mRNAs}$ between the 0 and $32 \mathrm{~h}$ group by qRT-PCR. Our results showed that the LINC00667:6 and lnc-HMGN1-1:12 were both up-regulated at $32 \mathrm{~h}$, compared 

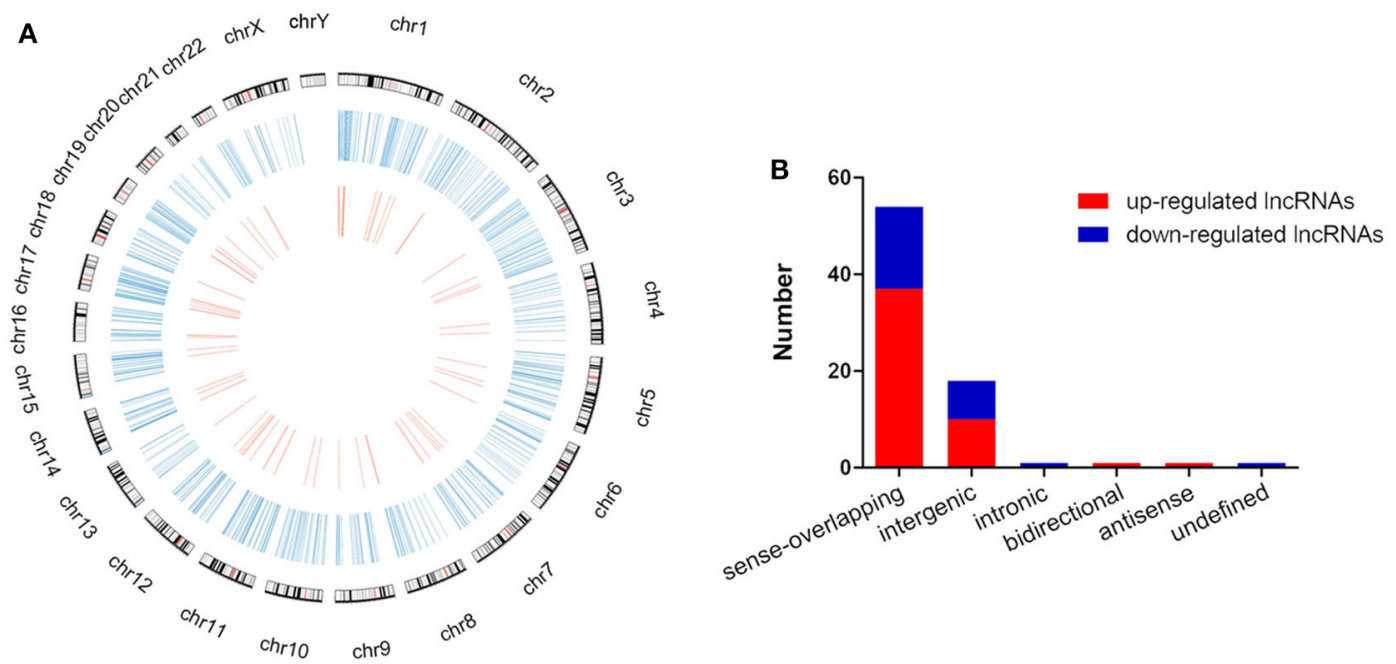

FIGURE 2 | Distribution and classification of the common differentially expressed IncRNAs. (A) The circos plot showed the distribution of IncRNAs on human chromosomes. The outermost layer of circos plot was chromosome map of the human genome (the black and white bars were chromosome cytobands, the red bars represented centromeres). The larger inner circle (blue) represented all differentially expressed IncRNAs at different time points, and the smaller inner circle (red) indicated the 76 common differentially expressed IncRNAs with fold change $>2.0, p<0.05$ and FDR $<0.05$. (B) These 76 common differentially expressed IncRNAs were classified into 6 types, including sense-overlapping, intergenic, intronic, bidirectional, antisense and undefined.

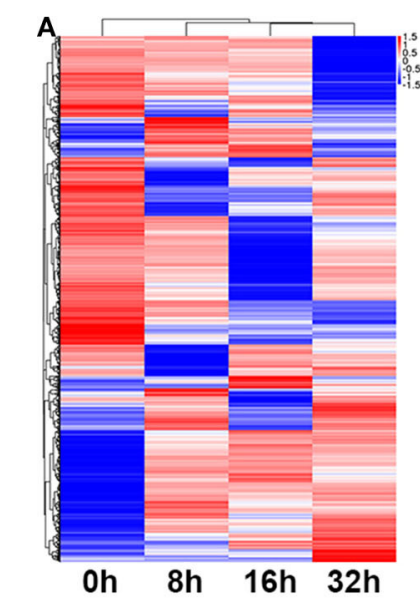

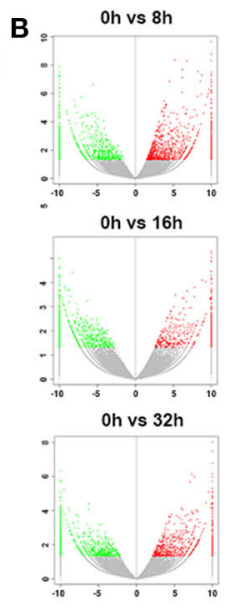

- Up-regulated

- Not significant

- Down-regulated

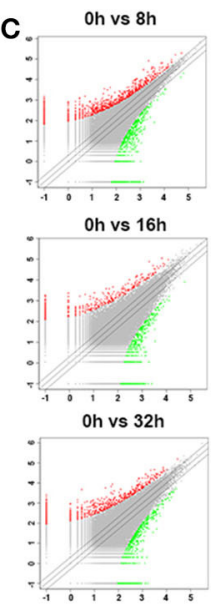

- Up-regulated

Not significant

Down-regulated

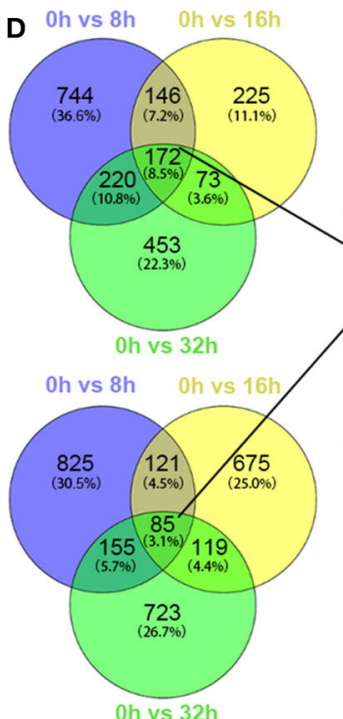

E

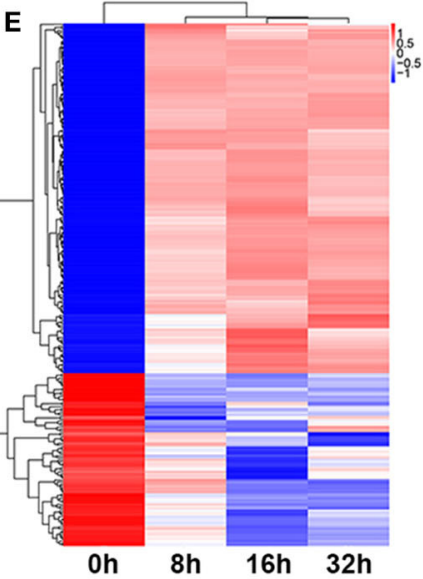
FIGURE 3 | Differentially expressed mRNA profile by sequencing. (A) The cluster heatmap showed all differentially expressed mRNAs at different time points (0, 8 , 16 ,
and $32 \mathrm{~h}) .(\mathbf{B}, \mathbf{C})$ The volcano and scatter plots presented differentially expressed mRNAs between different compared groups $(0$ vs. $8 \mathrm{~h}, 0$ vs. $16 \mathrm{~h}, 0$ vs. $32 \mathrm{~h})$, respectively. (D,E) Venn analysis analyzed the common differentially expressed mRNAs among the three compared groups, and described them as a cluster heatmap.

to the $0 \mathrm{~h}$ group, whereas other $3 \operatorname{lncRNAs}$ (lnc-LRRC24-2:1, lncAC007952.1.1-3:1, and lnc-CCNB1IP1-1:2) were down-regulated (Figure 4A, $\left.{ }^{*} P<0.05\right)$. Meanwhile, we also chose 5 random cancer-related genes for mRNA detection, and found that GADD45A, HBP1, and SESN2 were significantly up-regulated, whereas KIF20A and TOP2A were down-regulated, compared to the 0 h group (Figure $4 \mathrm{~B},{ }^{*} P<0.05$ ). These data were consistent well with the sequencing data, which demonstrated the high reliability and validity of the sequencing expression results.

\section{GO and KEGG Pathway Analysis}

According to the common differentially expressed mRNAs among the three compared groups $(0$ vs. $8 \mathrm{~h}, 0$ vs. $16 \mathrm{~h}$, 0 vs. $32 \mathrm{~h}$ ), the GO biological processes classification was 

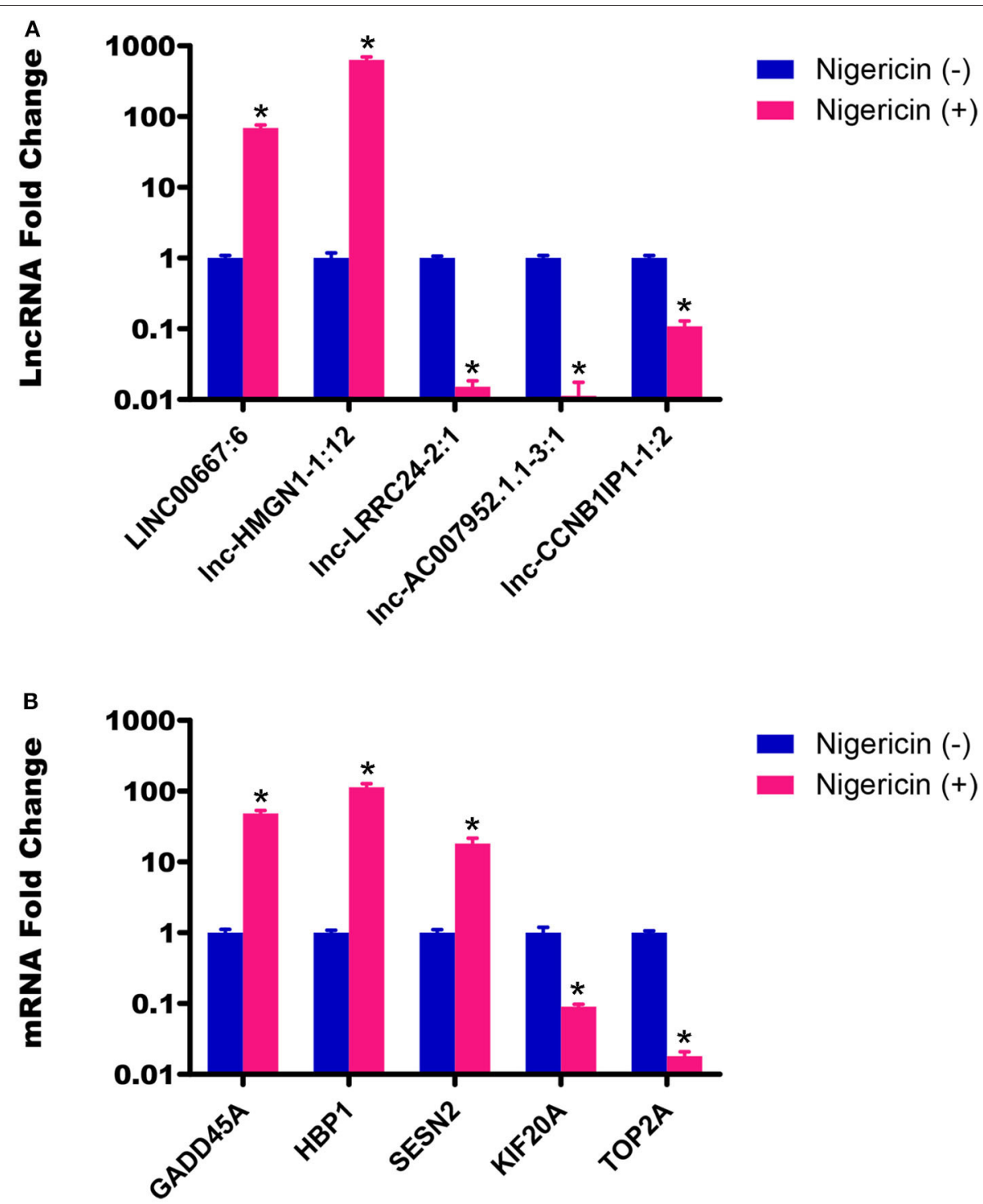

FIGURE 4 | Validation of sequencing data by qRT-PCR. (A) The expressions of 5 IncRNAs between the 0 and $32 \mathrm{~h}$ group were detected by qRT-PCR. (B) The expressions of $5 \mathrm{mRNAs}$ between the 0 and $32 \mathrm{~h}$ group were also determined by qRT-PCR $\left.{ }^{\star} P<0.05\right)$.

calculated. The top $10 \mathrm{GO}$ biological processes such as uridine catabolic process, nucleotide catabolic process and regulation of interleukin-6 biosynthetic process were involved in the nigericin damage. Meanwhile, the top 10 cellular components and molecular functions were also analyzed and presented in Figure 5A. KEGG pathway analysis for the common differentially expressed mRNAs was used to elucidate the pathways related to these mRNAs. Our data showed that differentially expressed mRNAs were significantly enriched in top 20 KEGG signaling pathways, including Aldosteroneregulated sodium reabsorption, Circadian rhythm, Mismatch repair, Drug metabolism-other enzymes, TNF signaling pathway, Transcriptional misregulation in cancers, TGF-beta signaling pathway, PI3K-Akt signaling pathway and so on (Figure 5B).
The corresponding $p$-value and enrichment score of the top 20 enrichment pathways were shown in Figure 5C. Using the results of KEGG enrichment analysis of genes, the network between all KEGG pathways and their corresponding genes was analyzed. One hundred sixty-nine pathways and 94 genes were included, and some genes involved in multiple KEGG pathways could be found to provide auxiliary reference for selection of candidate genes. For instance, PRKCA was found to participate in 60 KEGG pathways, including mTOR signaling pathway, PI3K-Akt signaling pathway, MicroRNAs in cancer, Choline metabolism in cancer, Wnt signaling pathway, MAPK signaling pathway, Pancreatic secretion, VEGF signaling pathway, Ras signaling pathway, Pathways in cancer and so on (Figure 6). 


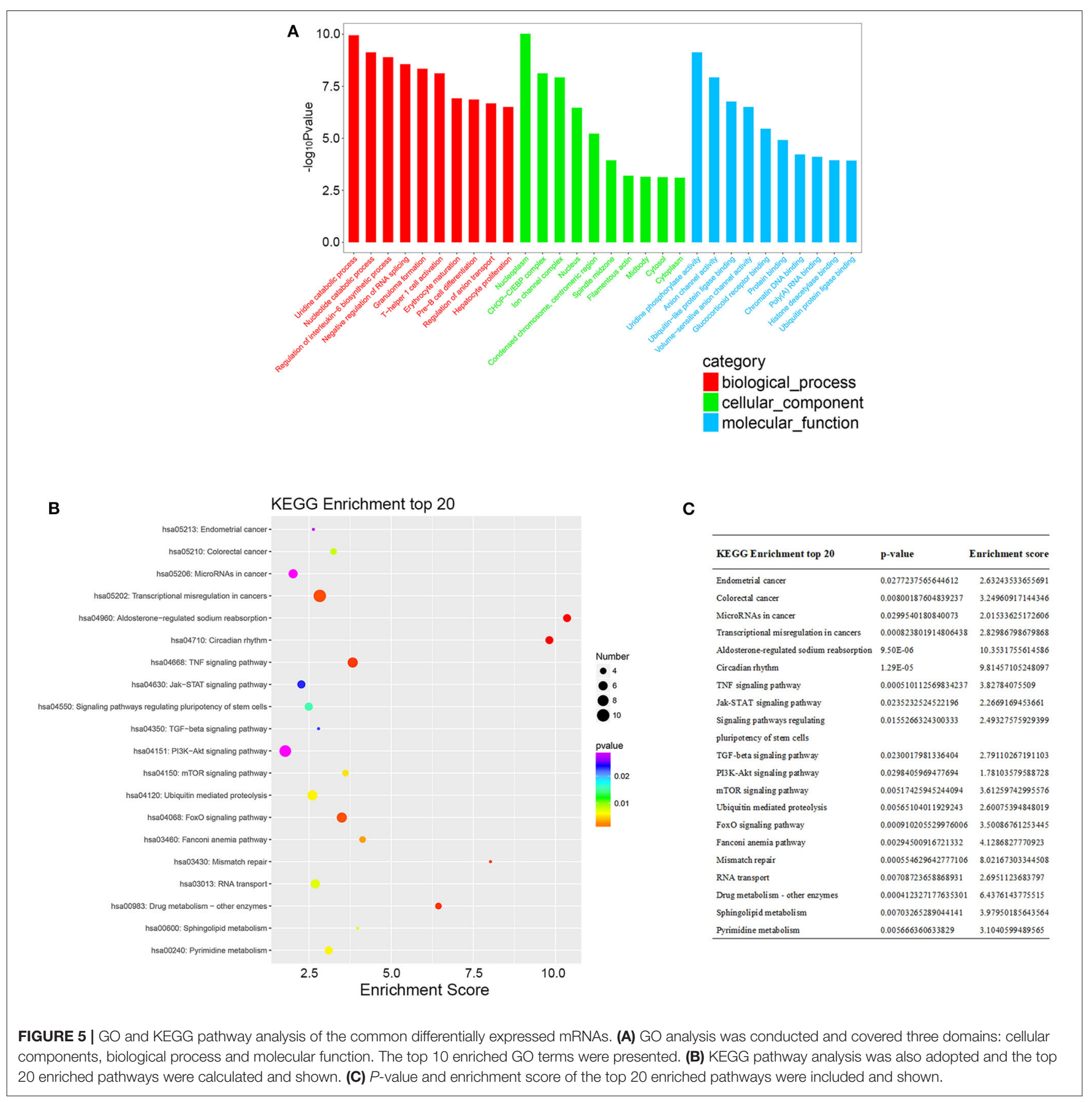

\section{Construction of Coding and Non-coding Co-expression Network}

Common differentially expressed mRNAs (five up-regulated and five down-regulated ones, respectively), which were proved to implicate in multiple biological processes including cell cycle, apoptosis, angiogenesis and metastasis, were selected to build this network (Figure 7). The network implied a complex relationship that one gene could correlate with multiple lncRNAs and one lncRNA might also regulate numerous mRNAs in different ways. As shown in Figure 7, up-regulated lnc-AGRN2_9 was positively correlated with HBP1, GADD45A, SIK1, and SESN2, and negatively associated with TOP2A, CKAP2, while these mRNAs were implicated in apoptosis. Meanwhile, downregulated SSX2IP, which was involved in tumorigenesis and 


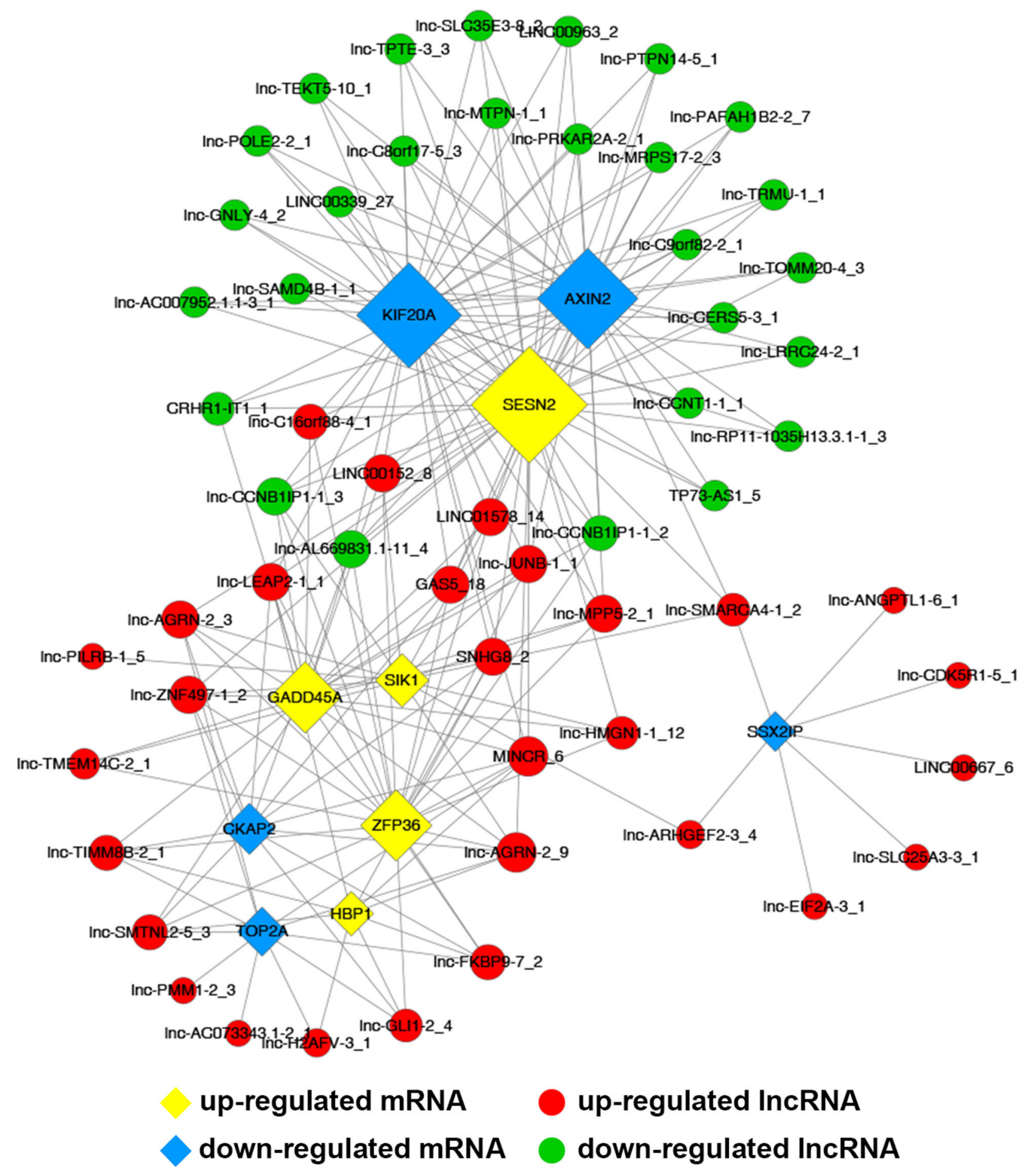

FIGURE 6 | The network between KEGG pathways and their corresponding genes was analyzed, in which 169 pathways and 94 genes were included.

metastasis, was negatively correlated with lnc-SMARCA4-1_2, lnc-EIF2A-3_1, lnc-SLC25A3-3_1, lnc-ANGPTL1-6_1, lnc-ARHGEF2-3_4, lnc-CDK5R1-5_1, and LINC00667_6.
The co-expression network might imply the potential regulatory mechanisms between lncRNAs and mRNAs in the nigericin anti-cancer process. 


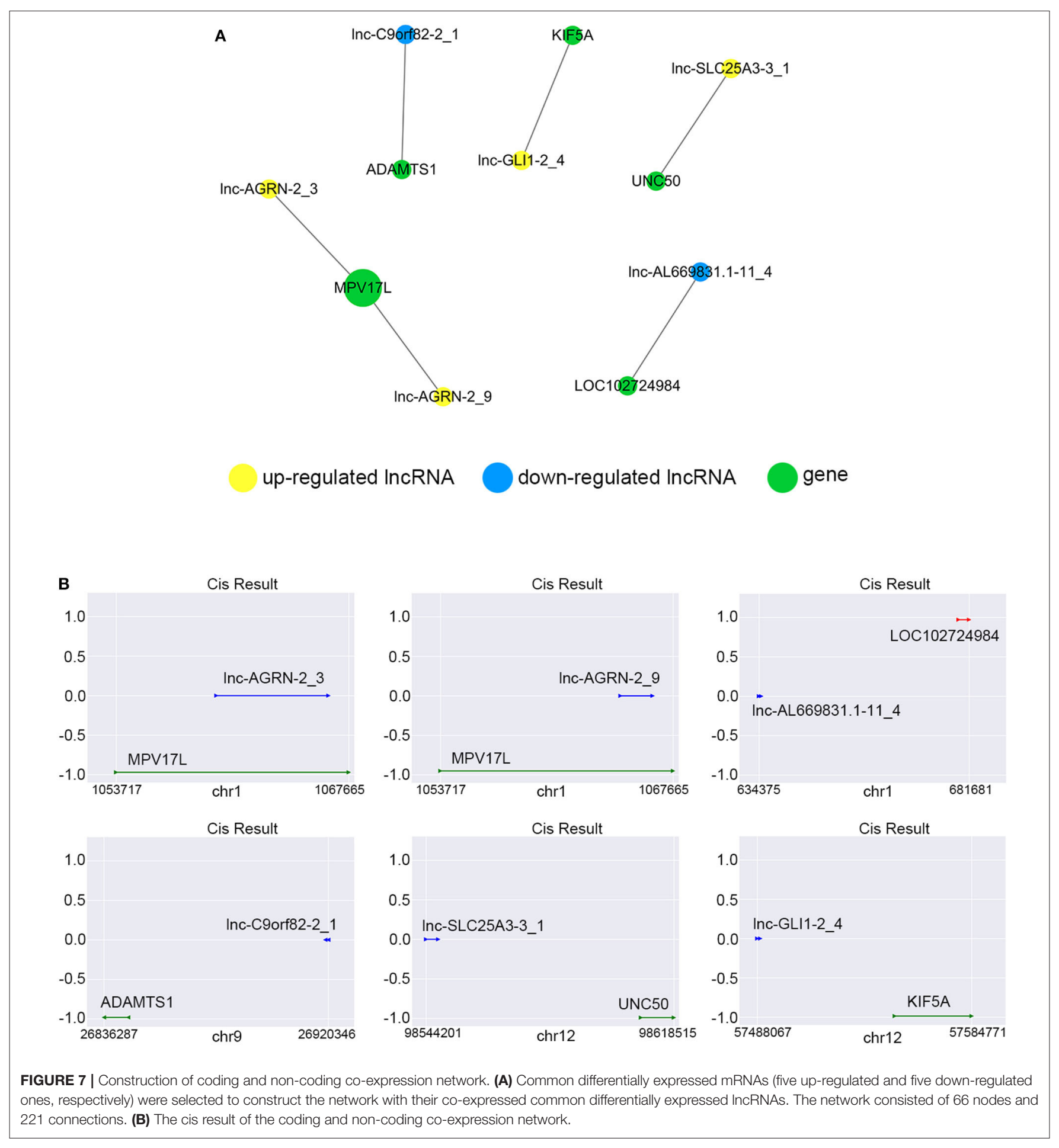

\section{Cis-Regulating Function Prediction of IncRNAs}

We constructed the correlated expression networks to elucidate the relationship between the common differentially expressed lncRNAs and their co-expressed adjacent coding genes. Among all the 76 common differentially expressed lncRNAs, only 6 lncRNAs were found to own neighboring protein-coding genes, and these 6 lncRNAs' potential cis-regulation networks were described in Figure 8A. However, each lncRNA had only one nearby coding gene. For example, lnc-AGRN-2_3 and 
MPV17L, lnc-AGRN-2_9 and MPV17L, lnc-AL669831.1-11_4 and LOC102724984, lnc-C9orf82-2_1 and ADAMTS1, lnc-GLI12_4 and KIF5A, lnc-SLC25A3-3_1 and UNC50 were shown in Figure 8B. The networks might furnish valuable clue for these lncRNAs with nearby coding genes.

\section{Trans-Regulation of IncRNAs (TF-IncRNA and TF-IncRNA-Gene Network)}

Despite the prevalence of lncRNA-mediated cis-regulation, examples of trans-acting lncRNAs have also been reported $(25,26)$. For trans-regulation prediction, we constructed a coexpression network combined by these common differentially expressed lncRNAs with TFs. With a threshold of $P<0.01$ and FDR $<0.01$, the top 200 closest relationships were selected, while we constructed a TF-lncRNA binary network. The network showed that 44 up-regulated lncRNAs were found to correspond to $31 \mathrm{TFs}$, and 27 down-regulated lncRNAs corresponded to 12 TFs (Figures 9A,B). Moreover, we introduced target genes to build TF-lncRNA-gene ternary network. 10 up-regulated lncRNAs correspond to 3 TFs and 283 target genes, while six down-regulated lncRNAs were found to associate with 3 TFs and 125 target genes (Figure 10). Interestingly, up to 14 dys-regulated lncRNAs were regulated by 5 TFs, such as MYC, TAF1, E2F4, STAT1, and STAT2. The results implied that these TFs might also participate in the nigericin anti-cancer damage.

\section{PPI Network Construction}

As shown in Figure 11, a total of 152 genes of the 257 common differentially expressed genes were filtered into the PPI network containing 152 nodes and 644 edges. The nodes with high degrees were defined as hub proteins in the PPI networks and degree $>10$ was set as the cut-off criterion. In this network, a total of 12 nodes were selected as hub proteins, including TOP2A, MYC, ANAPC1, FBXW7, KIF20A, MTOR, CREB1, EXO1, MELK, NEDD4L, RACGAP1, and HERC2. The most significant hub proteins were TOP2A (degree $=40)$ and MYC (degree $=21)$. This network exhibited the interactions among these genes which might play a significant role in the nigericin treatment.

\section{DISCUSSION}

Recently, the anti-cancer effect of nigericin has drawn increasing attentions, and its molecular mechanisms toward cancer cells were gradually discovered. A newly study by Yakisich et al. demonstrated that nigericin might be used in a co-therapy model of lung cancer in combination with other chemotherapeutic agents (27). Coincidentally, our lab also implied that $\mathrm{Wnt} / \beta$ catenin signaling might have an essential role in colorectal cancer progression, and nigericin exerted anti-cancer effects on colorectal cancer cells by directly targeting the $\beta$-catenin destruction complex (28). Furthermore, our recent study has proved the potential toxicity of nigericin on human PC, and revealed the molecular mechanism of nigericin toward PC cells from the perspective of circRNA (23). However, the knowledge of nigericin needs to be further elucidated from multiple perspectives.
Along with the deepening of research on PC, numerous lncRNAs have shown to be essential for the tumorigenesis and progression by serving as tumor oncogenes or suppressors. In 2016, Li et al. found that long non-coding RNA metastasisassociated lung adenocarcinoma transcript 1 (MALAT1) could facilitate the advanced progression of PC by promoting autophagy in vitro (29). lncRNA myocardial infarctionassociated transcript (MIAT) was found remarkably increased in PC tissues and cell lines, and PC patients with high MIAT levels had poor prognosis than those with low MITA levels (30). In contrast, Lnc-PCTST might exhibit as a potential tumor suppressor in PC, which inhibited cell proliferation, invasion, tumorigenesis and EMT by modulating TACC-3 (31). To further explore the anti-cancer mechanism of nigericin, we used highthroughput and bioinformatics methods to predict the changes of coding and non-coding RNAs when cells were exposed to the drug.

Firstly, the global expression profile of lncRNAs and mRNAs for four different nigericin-treated time points was determined by a custom sequencing platform. By venn analysis, our data confirmed that 76 common dys-regulated lncRNAs including 49 up-regulated and 27 down-regulated ones might participate in the process of nigericin damage. These lncRNAs were widely distributed on all chromosomes except for sex chromosome X. Meanwhile, the common differentially expressed mRNAs among the 3 compared groups were also found, in which 172 mRNAs were common up-regulated and 85 ones were down-regulated. Subsequently, we chose 5 random $\operatorname{lncRNAs}$ and 5 cancer-related genes for PCR detection between the 0 and $32 \mathrm{~h}$ group. The data were consistent well with our sequencing data, which demonstrated the high reliability and validity of the sequencing expression results. Of these common differentially expressed mRNAs, GADD45A was found to be variously expressed in cell lines derived from PC, and adenoviral-mediated expression of GADD45A (Ad-G45a) in these cells resulted in apoptosis via caspase activation and cell-cycle arrest in the G2/M phase (32). HMG-box transcription factor 1 (HBP1) had been described as a negative regulator of the $\mathrm{Wnt} / \beta$-catenin signaling in many cancers, including breast cancer (33), osteosarcoma (34), glioma (35), and colorectal carcinoma (36). A recent study by Chan also indicated that HBP1 acted as a direct downstream target of FOXO1, and potently suppressed the phenotypes of oral cancer (37). Besides, other 3 validated genes (SESN2, SIK1, and KIF20A) were also proved to influence the proliferation, migration and invasion of PC cells (38-41). These results might provide clues to the potential mechanisms of nigericin in PC.

Next, we conducted GO and KEGG pathway analyses to uncover the roles of these common differentially expressed mRNAs after nigericin treatment. The top 10 GO biological processes such as uridine catabolic process, nucleotide catabolic process and regulation of interleukin- 6 biosynthetic process were found in the nigericin damage. Meanwhile, the differentially expressed mRNAs were significantly enriched in top 20 KEGG signaling pathways, including Aldosterone-regulated sodium reabsorption, Circadian rhythm, Mismatch repair, Drug metabolism-other enzymes, TNF signaling pathway, Transcriptional misregulation in cancers, TGF-beta signaling 

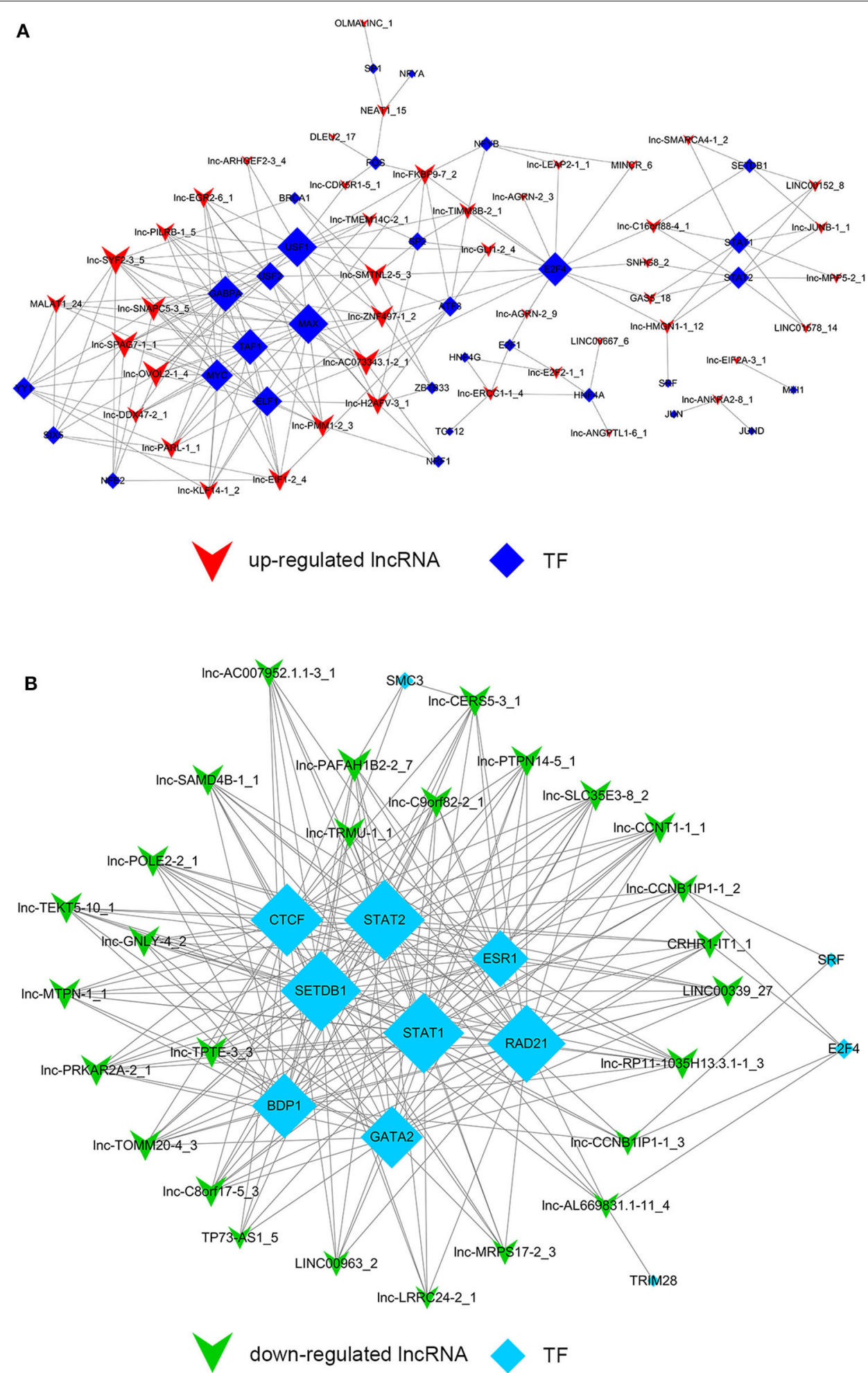

FIGURE 8 | Cis-regulation prediction of IncRNAs. (A) Among the 76 common differentially expressed IncRNAs, 6 IncRNAs were found to own neighboring protein-coding genes coding genes. The potential cis-regulation network was described. (B) The location and distances between IncRNAs and their nearby coding genes on the chromosome were presented. 


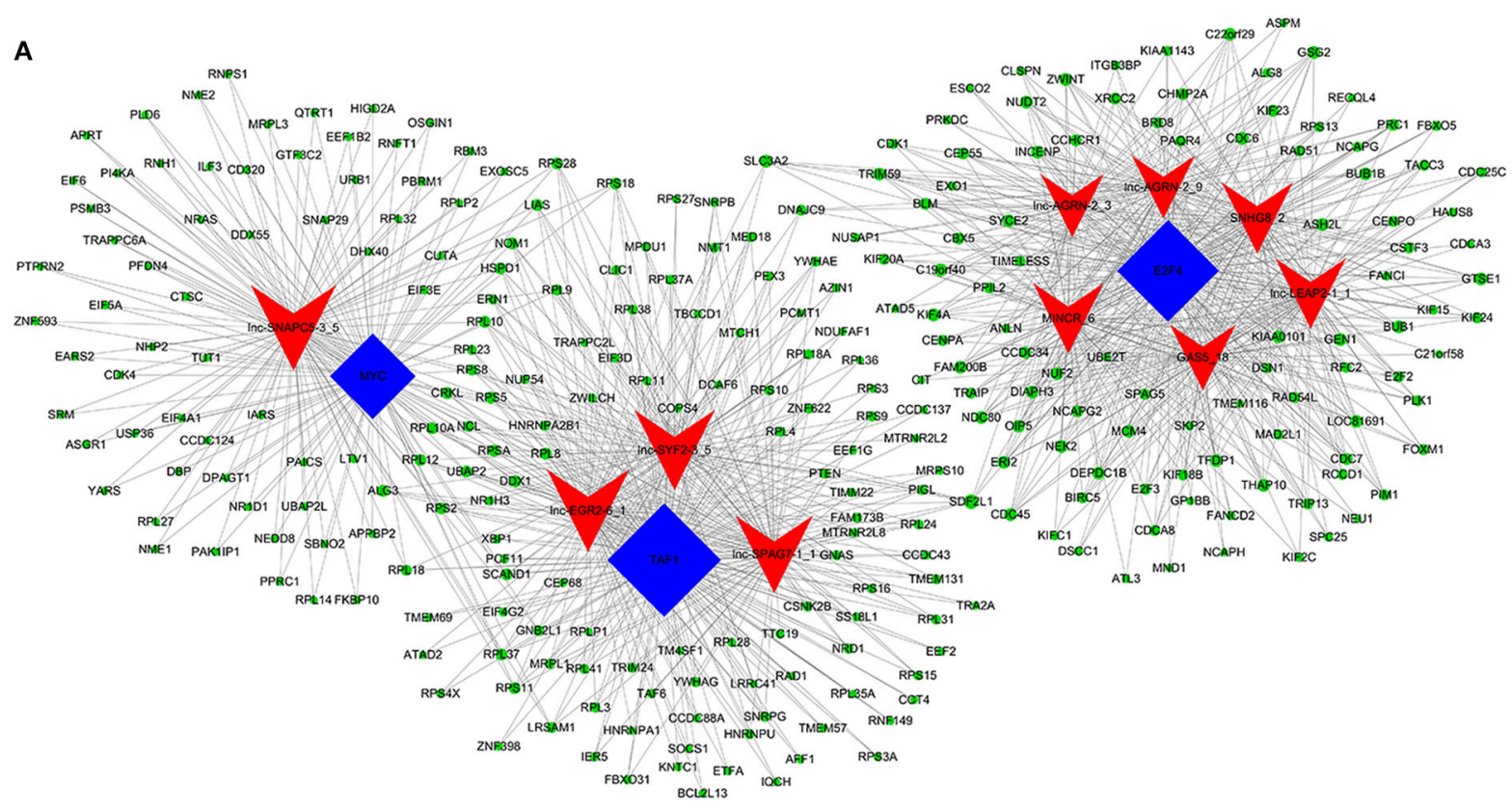

$\checkmark$ up-regulated IncRNA $>$ TF $\bigcirc$ gene

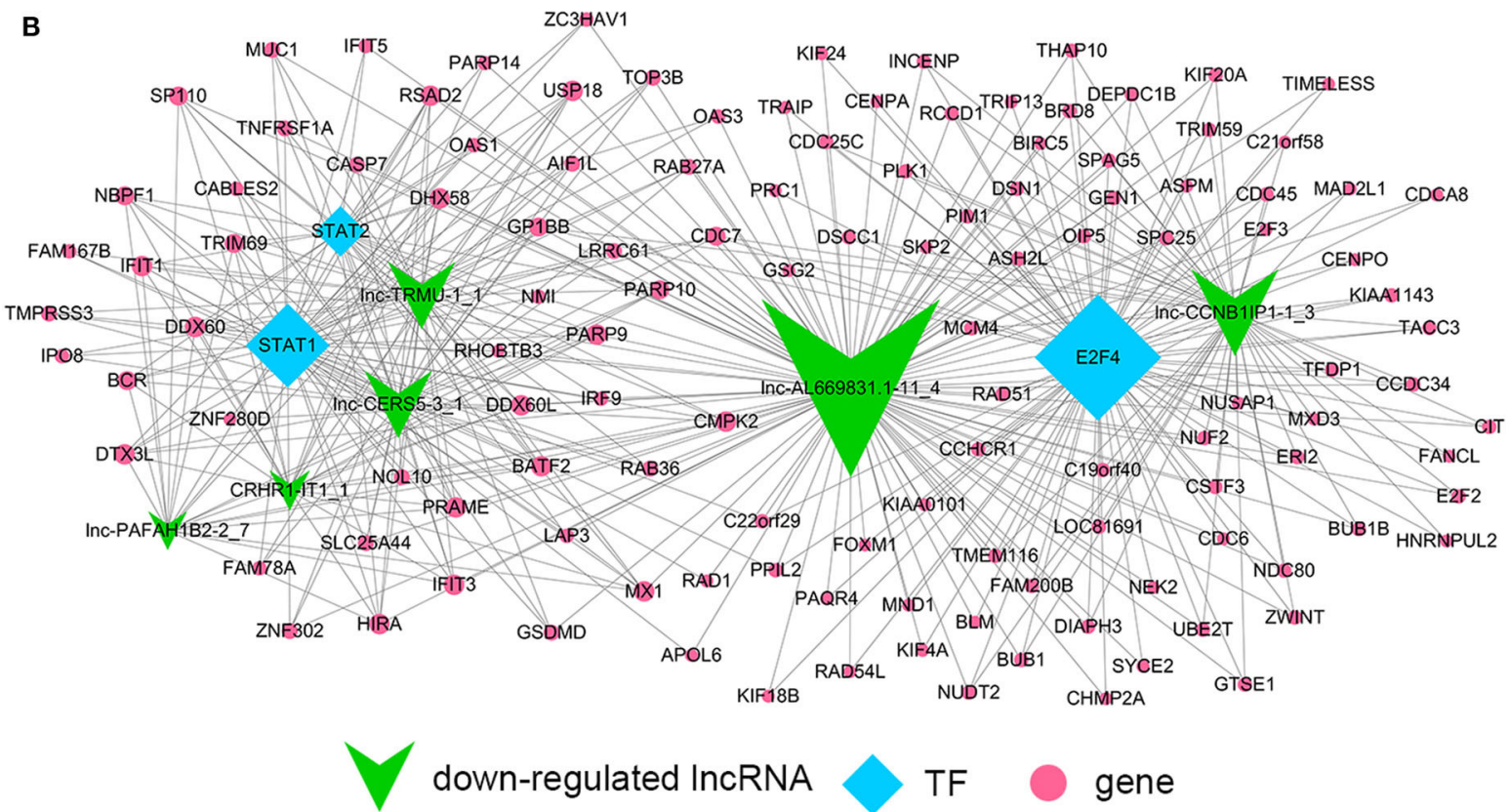

FIGURE 9 | Trans-regulation prediction of IncRNAs (TF-IncRNA binary network). (A) Among the 76 common differentially expressed IncRNAs, 44 up-regulated IncRNAs were found to correspond to 31 TFs. (B) 27 down-regulated IncRNAs corresponded to 12 TFs. 


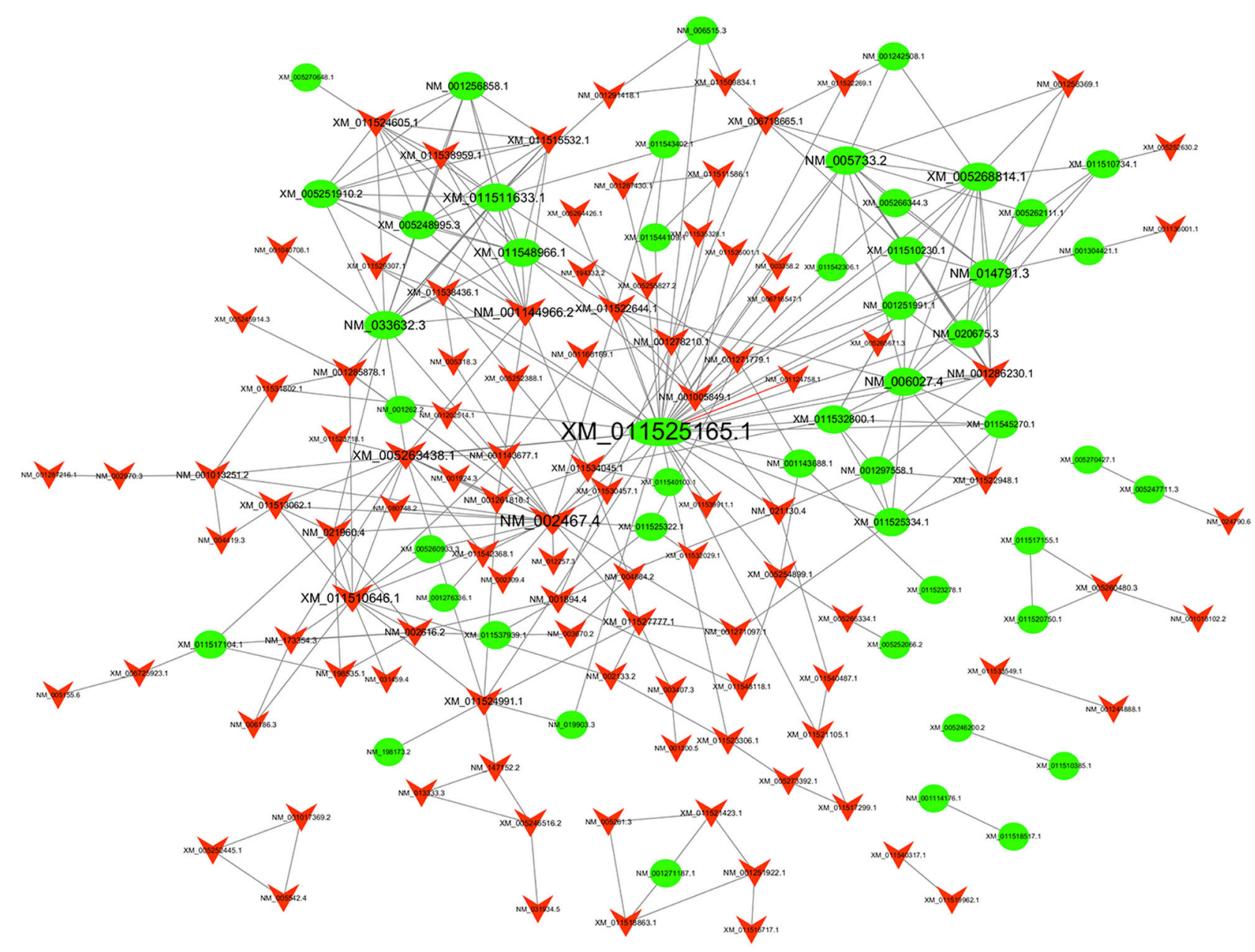

up-regulated mRNA $\bigcirc$ down-regulated mRNA

FIGURE 10 | Trans-regulation prediction of IncRNAs (TF-IncRNA-gene ternary network). Target genes were introduced to build TF-IncRNA-gene ternary network. 10 up-regulated IncRNAs correspond to 3 TFs and 283 target genes, while 6 down-regulated IncRNAs were found to associate with 3 TFs and 125 target genes.

pathway, PI3K-Akt signaling pathway and so on. Moreover, the network between all KEGG pathways and their corresponding genes was also analyzed. These nigericin-related pathways have been also reported in PC. For example, the PI3K/Akt signaling pathway is related with PC metastasis. Tanno et al. showed that increased insulin-like growth factor I receptor expression induced by active Akt markedly enhanced the invasiveness of human PC cells (42). A recent review from Murthy et al. also described the role of PI3K signaling in PC development and progression (43). In 2014, Zhu et al. provided valuable baseline information regarding the TGF- $\beta$ pathway in PC, which could be utilized in targeted therapy clinical trials (44). These involved non-coding RNAs (lncRNAs and mRNAs) and GO/KEGG analyses might partly explain the phenomena that nigericin had the anti-cancer properties.

To better understand the mechanisms of nigericin in PC cells, we built the co-expression network between lncRNAs and mRNAs. The network implied a complex relationship that one gene could correlate with multiple lncRNAs and one lncRNA might also regulate numerous mRNAs in different ways. For instance, up-regulated lnc-AGRN-2_9 was positively correlated with HBP1, GADD45A, SIK1, and SESN2, and negatively associated with TOP2A, CKAP2, while these mRNAs were implicated in tumorigenesis $(32,37-41,45)$. The coexpression network might imply the potential regulatory mechanisms between lncRNAs and mRNAs in the nigericin anti-cancer process.

It has been known that lncRNAs can cis-regulate the coexpressed and nearby coding genes (24). In this study, we constructed a cis-regulated network with the criterion that coding genes located at $100 \mathrm{k}$ bp upstream and downstream of lncRNAs on the chromosome. Our results showed that 6 of 76 common differentially expressed lncRNAs possessed cis-regulated genes, and each of the 6 lncRNAs only had 


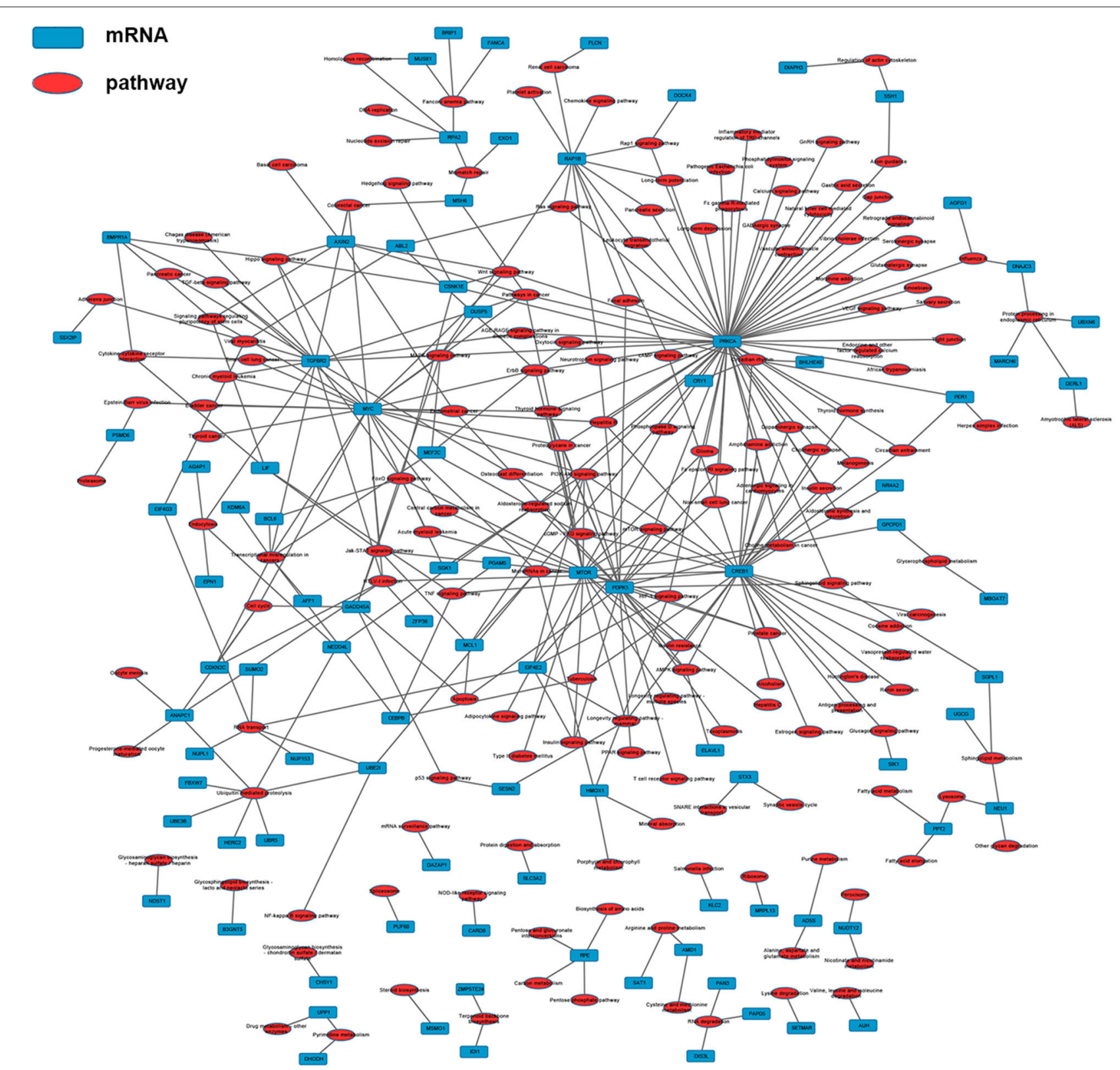

FIGURE 11 | Protein-protein interaction (PPI) network. A total of 152 common differentially expressed genes (103 up-regulated genes and 49 down-regulated ones) were filtered into the network complex. The lines represented the interaction relationship among these genes.

one neighboring protein-coding gene. For example, we found that lnc-AGRN-2_3 and lnc-AGRN-2_9 shared the same cisregulated gene $M P V 17 \mathrm{~L}$, which indicated that these two lncRNAs might play a similar role. Lnc-C9orf82-2_1 cisregulated ADAMTS1, and Masui et al. also suggested that ADAMTS1 was a potential biomarker to detect early-stage PCs (46). UNC50 has long been recognized as a Golgi apparatus protein in yeast, and is involved in nicotinic receptor trafficking in Caenorhabditis elegans. In 2015, Fang et al. found that UNC50 was correlated with G1/S transition and proliferation in hepatocellular carcinoma via the influencing epidermal growth factor receptor trafficking (47). Interestingly, our data showed that UNC50 was involved with the nigericin damage, which could be cis-regulated by lnc-SLC25A3-3_1. These results revealed the prevalence of lncRNA-mediated cis-regulations on nearby genes during the nigericin damage.

On the other hand, previous reports have indicated that lncRNAs are capable of binding to a specific site or sequence, including $\mathrm{TFs}$, to achieve trans-regulation functions. We constructed a TF-lncRNA binary network combined by these common differentially expressed lncRNAs with TFs. The network showed that 44 up-regulated lncRNAs were found to correspond 
to 31 TFs, and 27 down-regulated lncRNAs corresponded to 12 TFs. Furthermore, we introduced target genes to build TF-lncRNA-gene ternary network. 10 up-regulated lncRNAs correspond to 3 TFs and 283 target genes, while 6 down-regulated lncRNAs were found to associate with 3 TFs and 125 target genes. Interestingly, up to 14 dys-regulated IncRNAs were regulated by 5 TFs, such as MYC, TAF1, E2F4, STAT1, and STAT2. Recent evidence strongly suggests that these 5 TFs potentially regulate the expression of target genes in PC or other cancers. For instance, Valenti et al. found that Mutp53 and E2F4 proteins formed a transcriptional repressive complex that assembled onto the regulatory regions of BRCA1 and RAD17 genes inhibiting their expressions in head and neck squamous cell carcinoma (48). Guerrero-Zotano et al. identified 18 of the 20 E2F4 target genes, and suggested a potential benefit of adjuvant CDK4/6 inhibitors in patients with $\mathrm{ER}^{+}$breast cancer who failed to respond to preoperative estrogen deprivation (49). STAT1, which is a member of the family of signal transducers and transcription activators, corresponded to lymph node metastasis, advanced stage, tumor dedifferentiation and poor prognosis in patients with PC (50). A study from Seshacharyulu et al. also confirmed STAT1 as a key regulator through down-regualtion of MUC4 in PC (51). Thus, our cis- and trans-regulation predictions might provide a deep insight into the involved lncRNAs in nigericin treatment.

Finally, a PPI network with common differentially expressed genes, in which 12 hub proteins were identified, including TOP2A, MYC, ANAPC1, FBXW7, KIF20A, MTOR, CREB1, EXO1, MELK, NEDD4L, RACGAP1, and HERC2. The most significant hub proteins were TOP2A and MYC. TOP2A could induce tumor development and progression in many cancer types, including PC (52), prostate cancer (53) and breast cancer (54). In 2016, a phase II study by Tarpgaard et al. found that metastatic colorectal cancer (mCRC) patients, who were refractory to treatment with oxaliplatin-based chemotherapy, had TOP2A gene amplification in their tumor cells (55). Similarly, human estrogen receptor-positive breast cancer cells typically displayed elevated levels of $\mathrm{Myc}$ protein due to overexpression of MYC mRNA (56). Other studies had also identified the abnormal expression of MYC-binding protein (MYCBP) during tumorigenesis in multiple types of cancer, such as gastric cancer (57), colon cancer (58), and PC (59). Therefore, this core PPI network exhibited the associations

\section{REFERENCES}

1. Benson $A B$ III. Adjuvant therapy for pancreatic cancer: one small step forward. JAMA. (2007) 297:311-3. doi: 10.1001/jama.297.3.311

2. Siegel R, Ma J, Zou Z, Jemal A. Cancer statistics, 2014. CA Cancer J Clin. (2014) 64:9-29. doi: 10.3322/caac. 21208

3. Laheru D, Jaffee EM. Immunotherapy for pancreatic cancer - science driving clinical progress. Nat Rev Cancer. (2005) 5:459-67. doi: 10.1038/nrc1630

4. Duffy MJ, Sturgeon C, Lamerz R, Haglund C, Holubec VL, Klapdor $\mathrm{R}$, et al. Tumor markers in pancreatic cancer: a European Group on Tumor Markers (EGTM) status report. Ann Oncol. (2010) 21:4417. doi: 10.1093/annonc/mdp332

5. Vincent A, Herman J, Schulick R, Hruban RH, Goggins M. Pancreatic cancer. Lancet. (2011) 378:607-20. doi: 10.1016/S0140-6736(10)62307-0 between these interested genes, which might provide useful clues for the mechanism analysis of nigericin in PC.

\section{CONCLUSION}

In summary, our experiments further investigated the anti-cancer properties of nigericin in PC. In light of the highthroughput RNA sequencing analysis, we comprehensively characterized the potential contributions of lncRNAs and mRNAs after nigericin exposure. Additionally, the bioinformatics analyses, including GO and KEGG analysis, coding and non-coding co-expression network, cis- and transregulation predictions and PPI network, were applied to annotate the potential regulatory mechanisms among these coding and non-coding RNAs during the nigericin anti-cancer process. Our data provided new insight into the molecular mechanism of nigericin toward cancer cells, and suggested a possible clinical application in PC.

\section{DATA AVAILABILITY STATEMENT}

The datasets presented in this study can be found in online repositories. The names of the repository/repositories and accession number(s) can be found below: the NCBI Sequence Read Archive (https://www.ncbi.nlm.nih.gov/sra, PRJNA543685).

\section{AUTHOR CONTRIBUTIONS}

ZX conceived the project and wrote the manuscript. QZ and YX reviewed the manuscript. All authors participated in experiment and data analysis.

\section{FUNDING}

This study was supported by grants from the National Science Foundation of China (81802340, 81902805), the Suzhou Gusu Medical Youth Talent (GSWS2019032), the Project of Invigorating Health Care through Science, Technology and Education, Jiangsu Provincial Medical Youth Talent (QNRC2016723) and National Natural Science Foundation of China (8180100710).

6. Pramanik KC, Fofaria NM, Gupta P, Ranjan A, Kim SH, Srivastava SK. Inhibition of $\beta$-catenin signaling suppresses pancreatic tumor growth by disrupting nuclear $\beta$-catenin/TCF-1 complex: critical role of STAT-3. Oncotarget. (2015) 6:11561-74. doi: 10.18632/oncotarget.3427

7. Gobbi PG, Federico M. What has happened to VBM (vinblastine, bleomycin, and methotrexate) chemotherapy for early-stage Hodgkin lymphoma? Crit Rev Oncol Hematol. (2012) 82:18-24. doi: 10.1016/j.critrevonc.2011.04.003

8. Altena R, Fehrmann RS, Boer H, de Vries EG, Meijer C, Gietema JA. Growth differentiation factor 15 (GDF-15) plasma levels increase during bleomycin- and cisplatin-based treatment of testicular cancer patients and relate to endothelial damage. PLoS ONE. (2015) 10:e0115372. doi: 10.1371/journal.pone.0115372

9. Kumar SM, Biswas S, Sarkar S, Mandal SS, Biswas J. Analytical study on the efficacy of neoadjuvant chemotherapy using a combination of methotrexate, 
bleomycin, and cisplatin in the management of advanced squamous cell Carcinoma of the buccal mucosa. Indian J Med Paediatr Oncol. (2017) 38:3458. doi: 10.4103/ijmpo.ijmpo_123_17

10. Zhi QM, Chen XH, Ji J, Zhang JN, Li JF, Cai Q, et al. Salinomycin can effectively kill ALDH(high) stem-like cells on gastric cancer. Biomed Pharmacother. (2011) 65:509-15. doi: 10.1016/j.biopha.2011.06.006

11. Zhou J, Li P, Xue XF, He SB, Kuang YT, Zhao H, et al. Salinomycin induces apoptosis in cisplatin-resistant colorectal cancer cells by accumulation of reactive oxygen species. Toxicol Lett. (2013) 222:139-45. doi: 10.1016/j.toxlet.2013.07.022

12. Yadav V, Varshney P, Sultana S, Yadav J, Saini N. Moxifloxacin and ciprofloxacin induces S-phase arrest and augments apoptotic effects of cisplatin in human pancreatic cancer cells via ERK activation. BMC Cancer. (2015) 15:581. doi: 10.1186/s12885-015-1560-y

13. Yadav V, Sultana S, Yadav J, Saini N. Gatifloxacin induces S and G2-phase cell cycle arrest in pancreatic cancer cells via p21/p27/p53. PLoS ONE. (2012) 7:e47796. doi: 10.1371/journal.pone.0047796

14. Shavit N, Dilley RA, San Pietro A. Ion translocation in isolated chloroplasts. uncoupling of photophosphorylation and translocation of $\mathrm{k}+$ and $\mathrm{h}+$ ions induced by nigericin. Biochemistry. (1968) 7:2356-63. doi: 10.1021/bi00846a043

15. Poole DT, Butler TC, Williams ME. The effects of nigericin, valinomycin, and 2,4-dinitrophenol on intracellular $\mathrm{pH}$, glycolysis, and $\mathrm{K}+$ concentration of Ehrlich ascites tumor cells. Biochim Biophys Acta. (1972) 266:46370. doi: 10.1016/0005-2736(72)90102-2

16. Mashima T, Okabe S, Seimiya H. Pharmacological targeting of constitutively active truncated androgen receptor by nigericin and suppression of hormonerefractory prostate cancer cell growth. Mol Pharmacol. (2010) 78:84654. doi: 10.1124/mol.110.064790

17. Deng CC, Liang Y, Wu MS, Feng FT, Hu WR, Chen LZ, et al. Nigericin selectively targets cancer stem cells in nasopharyngeal carcinoma. Int $J$ Biochem Cell Biol. (2013) 45:1997-2006. doi: 10.1016/j.biocel.2013.06.023

18. Zanke BW, Lee C, Arab S, Tannock IF. Death of tumor cells after intracellular acidification is dependent on stress-activated protein kinases (SAPK/JNK) pathway activation and cannot be inhibited by Bcl-2 expression or interleukin $1 \beta$-converting enzyme inhibition. Cancer Res. (1998) 58:2801-8.

19. Lu D, Choi MY, Yu J, Castro JE, Kipps TJ, Carson DA. Salinomycin inhibits Wnt signaling and selectively induces apoptosis in chronic lymphocytic leukemia cells. Proc Natl Acad Sci USA. (2011) 108:132537. doi: 10.1073/pnas.1110431108

20. Varnes ME, Bayne MT, Menegay HJ, Tuttle SW. Effect of the $\mathrm{K}+/ \mathrm{H}+$ ionophore nigericin on response of A549 cells to photodynamic therapy and tert-butylhydroperoxide. Free Radic Biol Med. (1993) 15:395-405. doi: 10.1016/0891-5849(93)90039-W

21. Vaupel P. Tumor microenvironmental physiology and its implications for radiation oncology. Semin Radiat Oncol. (2004) 14:198-206. doi: 10.1016/j.semradonc.2004.04.008

22. Zhou HM, Dong TT, Wang LL, Feng B, Zhao HC, Fan XK, et al. Suppression of colorectal cancer metastasis by nigericin through inhibition of epithelial-mesenchymal transition. World J Gastroenterol. (2012) 18:26408. doi: $10.3748 /$ wjg.v18.i21.2640

23. Xu ZH, Shen JQ, Hua SB, Wan DW, Chen Q, Han Y, et al. Highthroughput sequencing of circRNAs reveals novel insights into mechanisms of nigericin in pancreatic cancer. BMC Genomics. (2019) 20:716. doi: 10.1186/s12864-019-6032-3

24. Guenzl PM, Barlow DP. Macro lncRNAs: a new layer of cisregulatory information in the mammalian genome. RNA Biol. (2012) 9:731-41. doi: 10.4161/rna.19985

25. Hung T, Chang HY. Long noncoding RNA in genome regulation: prospects and mechanisms. RNA Biol. (2010) 7:582-5. doi: 10.4161/rna.7.5. 13216

26. Peng BY, Liu AF, Yu XW, Xu EW, Dai JB, Li MC, et al. Silencing of lncRNA AFAP1-AS1 suppressed lung cancer development by regulatory mechanism in cis and trans. Oncotarget. (2017) 8:93608-23. doi: 10.18632/oncotarget.20549

27. Yakisich JS, Azad N, Kaushik V, O'Doherty GA, Iyer AK. Nigericin decreases the viability of multidrug-resistant cancer cells and lung tumorspheres and potentiates the effects of cardiac glycosides. Tumour Biol. (2017) 39:1010428317694310. doi: $10.1177 / 1010428317694310$
28. Liu F, Li W, Hua SB, Han Y, Xu ZH, Wan DW, et al. Nigericin exerts anticancer effects on human colorectal cancer cells by inhibiting wnt/ $\beta$-catenin signaling pathway. Mol Cancer Ther. (2018) 17:952-65. doi: 10.1158/1535-7163.MCT-17-0906

29. Li L, Chen H, Gao Y, Wang YW, Zhang GQ, Pan SH, et al. Long noncoding RNA MALAT1 Promotes aggressive pancreatic cancer proliferation and metastasis via the stimulation of autophagy. Mol Cancer Ther. (2016) 15:223243. doi: 10.1158/1535-7163.MCT-16-0008

30. Li TF, Liu J, Fu SJ. The interaction of long non-coding RNA MIAT and miR133 play a role in the proliferation and metastasis of pancreatic carcinoma. Biomed Pharmacother. (2018) 104:145-50. doi: 10.1016/j.biopha.2018.05.043

31. Wang YD, Ding XY, Hu H, He Y, Lu ZP, Wu PF, et al. Long noncoding RNA lnc-PCTST predicts prognosis through inhibiting progression of pancreatic cancer by downregulation of TACC-3. Int J Cancer. (2018) 143:3143-54. doi: 10.1002/ijc.31657

32. Li YF, Qian HL, Li X, Wang HJ, Yu J, Liu YJ, et al. Adenoviral-mediated gene transfer of Gadd45a results in suppression by inducing apoptosis and cell cycle arrest in pancreatic cancer cell. J Gene Med. (2009) 11:313. doi: 10.1002 /jgm. 1270

33. Paulson KE, Rieger-Christ K, McDevitt MA, Kuperwasser C, Kim J, Unanue $\mathrm{V}$, et al. Alterations of the HBP1 transcriptional repressor are associated with invasive breast cancer. Cancer Res. (2007) 67:613645. doi: 10.1158/0008-5472.CAN-07-0567

34. Sun XH, Geng XL, Zhang J, Zhao HX, Liu Y. miR-155 promotes the growth of osteosarcoma in a HBP1-dependent mechanism. Mol Cell Biochem. (2015) 403:139-47. doi: 10.1007/s11010-015-2344-z

35. Yan ZY, Wang JP, Wang C, Jiao YB, Qi WG,s Che SS. miR-96/HBP1/Wnt/ $\beta$ catenin regulatory circuitry promotes glioma growth. FEBS Lett. (2014) 588:3038-46. doi: 10.1016/j.febslet.2014.06.017

36. Lee MF, Hsieh NT, Huang CY, Li CI. All trans-retinoic acid mediates MED28/HMG box-containing protein 1 (HBP1)/ $\beta$-catenin signaling in human colorectal cancer cells. J Cell Physiol. (2016) 231:1796-803. doi: $10.1002 /$ jcp. 25285

37. Chan CY, Huang SY, Sheu JJ, Roth MM, Chou IT, Lien CH, et al Transcription factor HBP1 is a direct anti-cancer target of transcription factor FOXO1 in invasive oral cancer. Oncotarget. (2017) 8:1453748. doi: 10.18632/oncotarget.14653

38. Donadelli M, Dalla Pozza E, Costanzo C, Scupoli MT, Piacentini P, Scarpa $A$, et al. Increased stability of P21(WAF1/CIP1) mRNA is required for ROS/ERK-dependent pancreatic adenocarcinoma cell growth inhibition by pyrrolidine dithiocarbamate. Biochim Biophys Acta. (2006) 1763:91726. doi: 10.1016/j.bbamcr.2006.05.015

39. Taniuchi K, Furihata M, Saibara T. KIF20A-mediated RNA granule transport system promotes the invasiveness of pancreatic cancer cells. Neoplasia. (2014) 16:1082-93. doi: 10.1016/j.neo.2014.10.007

40. Stangel D, Erkan M, Buchholz M, Gress T, Michalski C, Raulefs S, et al. Kif20a inhibition reduces migration and invasion of pancreatic cancer cells. J Surg Res. (2015) 197:91-100. doi: 10.1016/j.jss.2015.03.070

41. Pei YF, Yin XM, Liu XQ. TOP2A induces malignant character of pancreatic cancer through activating $\beta$-catenin signaling pathway. Biochim Biophys Acta. (2018) 1864:197-207. doi: 10.1016/j.bbadis.2017.10.019

42. Tanno S, Tanno S, Mitsuuchi Y, Altomare DA, Xiao GH, Testa JR. AKT activation up-regulates insulin-like growth factor I receptor expression and promotes invasiveness of human pancreatic cancer cells. Cancer Res. (2001) 61:589-93. doi: 10.1016/S0016-5085(08)80193X

43. Murthy D, Attri KS, Singh PK. Phosphoinositide 3-kinase signaling pathway in pancreatic ductal adenocarcinoma progression, pathogenesis, and therapeutics. Front Physiol. (2018) 9:335. doi: 10.3389/fphys.2018.00335

44. Javle M, Li Y, Tan DF, Dong XQ, Chang P, Kar S, et al. Biomarkers of TGF$\beta$ signaling pathway and prognosis of pancreatic cancer. PLOS ONE. (2014) 9:e85942. doi: 10.1371/journal.pone.0085942

45. Zhang R, Xu J, Zhao J, Bai JH. Proliferation and invasion of colon cancer cells are suppressed by knockdown of TOP2A. J Cell Biochem. (2018) 5:11320. doi: $10.1002 /$ jcb. 26916

46. Yi JM, Guzzetta AA, Bailey VI, Downing SR, Neste LV, Chiappinelli KB, et al. Novel methylation biomarker panel for the early detection of pancreatic cancer. Clin Cancer Res. (2013) 19:6544-55. doi: 10.1158/1078-0432.CCR-12-3224 
47. Fang Z, Zhou LN, Jiang SM, Cao LH, Yu L. UNC50 prompts G1/S transition and proliferation in HCC by regulation of epidermal growth factor receptor trafficking. PLoS ONE. (2015) 10:e0119338. doi: 10.1371/journal.pone.0119338

48. Valenti F, Ganci F, Fontemaggi G, Sacconi A, Strano S, Blandino G, et al. Gain of function mutant $\mathrm{p} 53$ proteins cooperate with E2F4 to transcriptionally downregulate RAD17 and BRCA1 gene expression. Oncotarget. (2015) 6:5547-66. doi: 10.18632/oncotarget.2587

49. Guerrero-Zotano AL, Stricker TP, Formisano L, Hutchinson KE, Stover DG, Lee $\mathrm{KM}$, et al. $\mathrm{ER}(+)$ breast cancers resistant to prolonged neoadjuvant letrozole exhibit an E2F4 Transcriptional program sensitive to CDK4/6 inhibitors. Clin Cancer Res. (2018) 24:2517-29. doi: 10.1158/1078-0432.CCR-17-2904

50. Sun Y, Yang SS, Sun N, Chen JQ. Differential expression of STAT1 and p21 proteins predicts pancreatic cancer progression and prognosis. Pancreas. (2014) 43:619-23. doi: 10.1097/MPA.0000000000000074

51. Kunigal S, Ponnusamy MP, Momi N, Batra SK, Chellappan SP. Nicotine, IFN$\gamma$ and retinoic acid mediated induction of MUC4 in pancreatic cancer requires E2F1 and STAT-1 transcription factors and utilize different signaling cascades. Mol Cancer. (2012) 11:24. doi: 10.1186/1476-4598-11-24

52. Tsiambas E, Karameris A, Tiniakos DG, Karakitsos P. Evaluation of topoisomerase IIa expression in pancreatic ductal adenocarcinoma: a pilot study using chromogenic in situ hybridization and immunohistochemistry on tissue microarrays. Pancreatology. (2007) 7:45-52. doi: 10.1159/000101877

53. de Resende MF, Vieira S, Chinen LT, Chiappelli F, Fonseca FP, Guimaraes GC, et al. Prognostication of prostate cancer based on TOP2A protein and gene assessment: TOP2A in prostate cancer. J Transl Med. (2013) 11:36. doi: 10.1186/1479-5876-11-36

54. An X, Xu F, Luo RZ, Zheng QF, Lu JB, Yang YH, et al. The prognostic significance of topoisomerase II alpha protein in early stage luminal breast cancer. BMC Cancer. (2018) 18:331. doi: 10.1186/s12885-018-4170-7
55. Tarpgaard LS, Qvortrup C, Nygard SB, Nielsen SL, Andersen DR, Jensen NF, et al. A phase II study of Epirubicin in oxaliplatinresistant patients with metastatic colorectal cancer and TOP2A gene amplification. BMC Cancer. (2016) 16:91. doi: 10.1186/s12885-0162124-5

56. Tian X, Chakrabarti A, Amirkhanov NV, Aruva MR, Zhang KJ, Mathew $B$, et al. External imaging of CCND1, MYC, and KRAS oncogene mRNAs with tumor-targeted radionuclide-PNA-peptide chimeras. Ann N Y Acad Sci. (2005) 1059:106-44. doi: 10.1196/annals.1339.038

57. Gong LJ, Xia YJ, Qian ZY, Shi J, Luo JG, Song GY, et al. Overexpression of MYC binding protein promotes invasion and migration in gastric cancer. Oncol Lett. (2018) 15:5243-9. doi: 10.3892/ol.2018.7944

58. Jung HC, Kim K. Identification of MYCBP as a $\beta$-catenin/LEF1 target using DNA microarray analysis. Life Sci. (2005) 77:1249-62. doi: 10.1016/j.lfs.2005.02.009

59. Hata T, Suenaga M, Marchionni L, Das AM, Yu J, Shindo K, et al. Genome-wide somatic copy number alterations and mutations in highgrade pancreatic intraepithelial neoplasia. Am J Pathol. (2018) 188:172333. doi: 10.1016/j.ajpath.2018.03.012

Conflict of Interest: The authors declare that the research was conducted in the absence of any commercial or financial relationships that could be construed as a potential conflict of interest.

Copyright $\odot 2020 \mathrm{Xu}$, Gao, Liu, Han, Dai, Wang, Wei, Kuang, Wan, Zhi and Xu. This is an open-access article distributed under the terms of the Creative Commons Attribution License (CC BY). The use, distribution or reproduction in other forums is permitted, provided the original author(s) and the copyright owner(s) are credited and that the original publication in this journal is cited, in accordance with accepted academic practice. No use, distribution or reproduction is permitted which does not comply with these terms. 